\title{
Novel copy-number variants in a population-based investigation of classic heterotaxy
}

\author{
Shannon L. Rigler, MD,2, Denise M. Kay, PhD³, Robert J. Sicko, BS ${ }^{3}$, Ruzong Fan, PhD', Aiyi Liu, PhD", \\ Michele Caggana, ScD, FACMG ${ }^{3}$, Marilyn L. Browne, PhD ${ }^{4,5}$, Charlotte M. Druschel, MD ${ }^{4,5}$, \\ Paul A. Romitti, PhD ${ }^{6}$, Lawrence C. Brody, PhD7 and James L. Mills, MD, MS'
}

Purpose: Heterotaxy is a clinically and genetically heterogeneous disorder. We investigated whether screening cases restricted to a classic phenotype would result in the discovery of novel, potentially causal copy-number variants.

Methods: We identified 77 cases of classic heterotaxy from all live births in New York State during 1998-2005. DNA extracted from each infant's newborn dried blood spot was genotyped with a microarray containing 2.5 million single-nucleotide polymorphisms. Copy-number variants were identified with PennCNV and cnvPartition software. Candidates were selected for follow-up if they were absent in unaffected controls, contained 10 or more consecutive probes, and had minimal overlap with variants published in the Database of Genomic Variants.
Results: We identified 20 rare copy-number variants including a deletion of $B M P 2$, which has been linked to laterality disorders in mice but not previously reported in humans. We also identified a large, terminal deletion of $10 \mathrm{q}$ and a microdeletion at $1 \mathrm{q} 23.1$ involving the MNDA gene; both are rare variants suspected to be associated with heterotaxy.

Conclusion: Our findings implicate rare copy-number variants in classic heterotaxy and highlight several candidate gene regions for further investigation. We also demonstrate the efficacy of copy-number variant genotyping in blood spots using microarrays.

Genet Med advance online publication 18 September 2014

Key Words: $B M P 2$ gene; congenital heart disease; copy-number variant; heterotaxy; MNDA gene

\section{INTRODUCTION}

Heterotaxy is defined as any abnormality of the internal organs in which their orientation is unclear with respect to one another. ${ }^{1}$ During normal embryonic development, lateralization results in a natural asymmetry of the internal organs. In heterotaxy, the lateralization process is disrupted and results in a wide range of abnormalities involving the cardiovascular, pulmonary, gastrointestinal, musculoskeletal, and central nervous systems. The spectrum of anomalies has been well characterized and previously reported by Zhu et al. ${ }^{2}$ in 2006. Heterotaxy may further be characterized by right or left isomerism, in which the paired and unpaired organs demonstrate symmetry or bilateral right/left sidedness. Classic heterotaxy is distinguished by its association with congenital heart disease (CHD), in addition to an unexpected arrangement of the other internal organs across the left-right axis of the body. ${ }^{2-4}$ Situs inversus totalis is excluded from the definition of heterotaxy because lateralization is maintained in a mirror-image reversal of the internal organs and it is not associated with CHD. ${ }^{1}$

Heterotaxy occurs in approximately 1 in 10,000 live births ${ }^{3}$ and accounts for approximately $3 \%$ of all $\mathrm{CHD}^{2}$ A $2: 1$ male-to-female predominance was reported in two large population-based studies. ${ }^{3,5}$ It has also been suggested that there is a higher prevalence in Asians ${ }^{6}$ and African Americans ${ }^{7}$ as compared with Caucasians. Although heterotaxy is rare, it is an important contributor to pediatric morbidity given that affected individuals have greater postsurgical complications and higher mortality rates than those with other types of $\mathrm{CHD}_{.}^{8,9}$

Most cases of heterotaxy are thought to be sporadic, although several genes have been implicated in human laterality disorders, including CFC1, NODAL, FOXH1, ACVR2B, LEFTY2, GDF1, CRELD1, NKX2-5, DNAH5, and DNAI1. ${ }^{10-17}$ Many of these genes are from the transforming growth factor- $\beta$ superfamily and have roles in the nodal signaling pathway. Other gene mutations have been shown to affect ciliary structure and function. X-linked mutations of ZIC3 account for the majority of familial cases and a small percentage of sporadic cases. ${ }^{4,18}$ Additional familial clusters suggest both autosomal dominant and autosomal recessive inheritance patterns. ${ }^{2,19}$ Known heterotaxy mutations account for less than $10-20 \%$ of all nonfamilial cases, suggesting that additional gene mutations exist. ${ }^{19,20}$

\footnotetext{
${ }^{1}$ Division of Intramural Population Health Research, Eunice Kennedy Shriver National Institute of Health and Human Development, National Institutes of Health, Department of Health and Human Services, Bethesda, Maryland, USA; ${ }^{2}$ Department of Neonatology, Walter Reed National Military Medical Center, Bethesda, Maryland, USA; ${ }^{3}$ Division of Genetics, Wadsworth Center, New York State Department of Health, Albany, New York, USA; ${ }^{4}$ Congenital Malformations Registry, New York State Department of Health, Albany, New York, USA; ${ }^{5}$ Department of Epidemiology and Biostatistics, University at Albany School of Public Health, Rensselaer, New York, USA; ${ }^{6}$ Department of Epidemiology, College of Public Health, The University of Iowa, Iowa City, Iowa, USA; ${ }^{7}$ Genome Technology Branch, National Human Genome Research Institute, National Institutes of Health, Department of Health and Human Services, Bethesda, Maryland, USA. Correspondence: Shannon L. Rigler (shannon.rigler@med.navy.mil)
} 
Animal studies have identified more than 80 genes involved in the development of the left-to-right axis; however, the majority of the genes that determine human left-to-right patterning have yet to be identified. ${ }^{2}$

Previous human studies examining the genetics of heterotaxy included cohorts with a wide spectrum of situs abnormalities including isolated CHD, isolated polysplenia or asplenia, and situs inversus totalis. ${ }^{4,11,13,20}$ Fakhro et al. ${ }^{20}$ analyzed 262 patients with heterotaxy, situs inversus totalis, and isolated transposition of the great arteries and found a high frequency of rare copy-number variants (CNVs), suggesting that CNVs may play a role in laterality disorders. The apparent genetic heterogeneity reported in that study and others like $\mathrm{it}^{10,11,13}$ may result from including cases with considerable phenotypic variation. We hypothesized that by using a more restricted definition of heterotaxy we would be more likely to identify recurring and potentially causal CNVs. It has been observed that $\sim 10 \%$ of patients with heterotaxy have close relatives affected by isolated CHD. ${ }^{2}$ Furthermore, several of the known heterotaxy mutations have also been identified in individuals with isolated $\mathrm{CHD}$, suggesting that their genetic etiologies may be related. ${ }^{21}$ This differs from other nonsyndromic laterality defects such as isolated malrotation of the bowel, asplenia, polysplenia, or renal defects, none of which have been linked to known heterotaxy gene mutations. As such, we predicted that classic heterotaxy, given its association with CHD, was more likely to be associated with mutations in fewer number of genes.

\section{MATERIALS AND METHODS}

\section{Cases}

Heterotaxy cases were selected from all live resident births in New York State (NYS) from 1998 to $2005(n=2,023,083)$. NYS requires reporting of congenital malformations occurring in the first 2 years of life to the NYS Congenital Malformations Registry (CMR). Diagnoses are made by community or hospital-based physicians and reported to the CMR; the diagnoses are then confirmed from supporting medical records. Trained coders convert diagnoses into British Pediatric Association codes, which are then cataloged in the CMR. Cases are reviewed by the medical director of the CMR for quality assurance. In the case of heterotaxy, a diagnosis of this complex would presumably be made by a pediatric cardiologist before coding using the various diagnostic capabilities of a tertiary medical facility, including echocardiogram, ultrasound, intraoperative reports, and autopsy. While complete medical records are not stored in the CMR, key narrative comments from the medical records are linked electronically to each case and are available for review.

To identify cases we searched for British Pediatric Association codes associated with any laterality disorder to include complete situs inversus, situs inversus with levocardia, situs ambiguus, situs inversus thoracis, situs inversus abdominis, asplenia, polysplenia, and dextrocardia with situs solitus (British Pediatric Association codes 746.800, 759.000-759.050, and 759.300759.390). We also searched narratives to ensure we captured any cases potentially not assigned a heterotaxy-associated
British Pediatric Association code. Eligible classic heterotaxy cases were defined as having both a major congenital heart defect and at least one additional abnormality of visceral situs. Isolated atrial septal defect or patent foramen ovale, patent ductus arteriosus, or peripheral pulmonic stenosis were not considered major congenital heart defects. Cases with dextrocardia or ventricular septal defects were included if they were associated with another anomaly of visceral situs but excluded if they had dextrocardia or ventricular septal defect as an isolated finding. To reduce the risk of identifying variants not related to classic heterotaxy, we excluded any cases that had a known chromosome abnormality or Mendelian gene syndrome, teratogenic syndrome, or another major congenital malformation not previously associated with heterotaxy. ${ }^{2}$ We also excluded any cases of primary ciliary dyskinesia because the phenotype or visceral orientation was not available in the CMR for this diagnosis. Isolated occurrences of situs inversus totalis, situs inversus thoracis, situs inversus abdominis, dextrocardia, polysplenia, and asplenia were excluded because they did not meet our definition of classic heterotaxy (see Supplementary Table S1 online for complete inclusion and exclusion criteria).

Cases were screened for point mutations in the most common heterotaxy genes available for clinical testing, including CFC1, ZIC3, FOXH1, ACVR2B, and NODAL, using a custom AmpliSeq panel (Life Technologies, Carlsbad, CA) on an Ion Torrent platform (for further details see Supplementary Materials and Methods, Supplementary Tables S2 and S3 online). The presence of a known heterotaxy mutation would result in exclusion from the $\mathrm{CNV}$ analysis but not from the population-based assessment. Demographic data were collected from the birth record when available, and variables were analyzed using Fisher's exact test or $t$-test, where applicable. Cases were assigned a random identification number, and all personally identifying data were removed before genotyping and analysis. The NYS Department of Health approved this study (IRB 07-007).

\section{Genotyping}

Seventy-four case and six quality control samples were genotyped. Three of 77 case samples could not be located and were not genotyped. For each sample, DNA was extracted from two 3-mm dried blood spot (DBS) punches using a laboratory-developed method. ${ }^{22}$ This resulted in approximately 200-1,200 ng of DNA per subject for array genotyping. Samples were genotyped at the Biomedical Genomics Center Core Facility at the University of Minnesota using Illumina HumanOmni2.5-8_v1 bead arrays and the Infinium HD assay protocol. Samples from six unaffected control subjects, including five DBS and one HapMap sample, were run in parallel as a measure of quality assurance. Data were analyzed using Illumina GenomeStudio v2011.1. The genotype no-call threshold was set at $<0.15$. Genotype clustering and data cleaning were carried out using two approaches. In the primary analysis, genotypes were called with reference to clusters based on the data generated in this project. In a secondary analysis, genotypes were clustered 
using the standard cluster file supplied by Illumina. For both analyses, genotypes and clusters were manually reviewed, reclustered, edited, and excluded (where appropriate) based on parameters and quality control metrics described in Illumina's Infinium Genotyping Data Analysis Technical Note (http:// res.illumina.com/documents/products/technotes/technote infinium_genotyping_data_analysis.pdf). A total of 2,291,202 and 2,299,719 autosomal markers were carried on to the CNV analysis from the primary and secondary genotyping analyses, respectively $(2,314,174$ total autosomal markers on the array). The mean sample call rate \pm SD (range) in the primary analysis was $99.4 \pm 0.6 \%$ (97.2-99.8), and the mean $\log R$ ratio deviation was $0.156 \pm 0.065(0.100-0.426)$. Compared with the primary analysis, mean call rates in the secondary analysis were lower $(97.2 \pm 2.6 \%$ (88.5-99.5)), and there was more deviation in the $\log R$ ratio $(0.379 \pm 0.088(0.192-0.694))$. Single-nucleotide polymorphism genotype reproducibility was $>99.99 \%$ for both analyses. One case, listed as female in the birth record but later genotyped as male, and was considered male for all analyses.

\section{CNV calling and annotation}

Autosomal CNVs were called using Illumina's cnvPartition algorithm version 3.1.6 and PennCNV version 2011/05/03. ${ }^{23}$ For both algorithms, data were GC wave adjusted, and the minimum number of probes required for a $\mathrm{CNV}$ call was three. The confidence threshold for CNV calling was set to the default value of 35 for cnvPartition and to 10 for PennCNV. CNV call files were annotated using custom $\mathrm{C}++$ scripts to (i) compare concordance of calls between analyses and algorithms; (ii) identify the proportion of each CNV that overlapped with CNVs from 53 studies cataloged in the Database of Genomic Variants (DGV2 beta); (iii) count the number of cases and controls carrying overlapping CNVs in the current study and in an additional group of 30 subjects genotyped by our lab; and (iv) identify intersecting transcripts and genes. ${ }^{24}$ Transcripts included full-length coding and noncoding transcripts with a well-characterized biotype downloaded from GENCODE (version 19, December 2013, accessed 14 May 2014). ${ }^{25}$ Genes were defined as those included in the Consensus CDS project (CCDS; release 15, accessed 14 May 2014). ${ }^{26}$ We reviewed CNV calls that had overlap with OMIM genes (accessed via the UCSC genome browser, 14 May 2014); genes linked to congenital heart defects $^{27}$; were involved in development or signal transduction (defined by reactome version 46, accessed 21 November 2013) ${ }^{28}$; were in pathogenic CNVs defined by the International Standards for Cytogenomic Arrays database (accessed via the UCSC genome browser, 14 May 2014); were associated with DECIPHER phenotypes (accessed 14 May 2014), including heterotaxy, heterotaxia, inversus, situs, or ambiguus; or if they were recently reported in heterotaxy cases. ${ }^{20}$

\section{CNV selection and prioritization}

$\mathrm{CNV}$ calls from our cases were compared against published genomic data on control subjects cataloged in DGVa/DGV2 ( $n=11,963$ individuals), which includes HapMap $(n=270$ individuals) and Children's Hospital of Philadelphia CNV $(n=2,026)$ data. CNVs were excluded from further investigation if they were also present in any of our control subjects (CNVs of the same type and with the same predicted breakpoints), contained fewer than 10 consecutive single-nucleotide polymorphism probes (to filter out smaller CNVs with fewer contributing probes that were less likely to be technically validated), or had substantial overlap with variants reported in DGVa/DGV2. Substantial overlap was defined as more than $50 \%$ of our CNV overlapping more than $50 \%$ of a DGVa/DGV2 variant. Coordinates from CNVs that were not excluded were then uploaded to DGVa using build37/hg19 coordinates and visually inspected for overlap with known CNVs. The CNV was excluded if it appeared similar to any previously published nonpathologic variant of the same type (i.e., deletion or duplication). Remaining CNVs were considered candidate heterotaxy-associated CNVs. Candidate CNVs were prioritized for follow-up validation studies if they were present in more than one subject, were biologically relevant based on the genes affected, or were previously found in association with heterotaxy.

\section{CNV validation}

Candidate CNVs surviving exclusion criteria were validated in the laboratory using two to four quantitative real-time polymerase chain reaction TaqMan assays (Applied Biosystems, Carlsbad, CA) per region on an ABI 7900HT system (details outlined in Supplemental Material and Methods online). All validated CNVs were subsequently screened against a total of 138 control samples from unaffected NYS births using at least one assay targeting the area of interest.

\section{RESULTS}

There were a total of 143 cases with any type of situs abnormality identified in the NYS CMR. Of these, 8 cases (5.5\%) were excluded for known or suspected genetic syndromes and 9 cases (6.3\%) were excluded for non-heterotaxy-associated malformations. Of the remaining cases, 77 met our case definition of classic heterotaxy: CHD with another abnormality of situs. Of the 77 eligible cases, 7 cases (9.1\%) were part of the National Birth Defects Prevention Study. In the NYS population, the birth prevalence of nonsyndromic classic heterotaxy was 1 in 26,000 births.

Demographic characteristics from classic heterotaxy cases were compared to a random sample of infants from NYS $(n=$ 7,683 ) with no known major birth defects (Table 1). We found that mothers of classic heterotaxy cases were more likely than mothers of controls to be Asian (14.3 vs. $6.8 \% ; P=0.0194$ ) or Hispanic (27.3 vs. $17.7 \%$; $P=0.0356$ ). Classic heterotaxy was also more common in children of mothers who were at the extremes of the prepregnancy body mass index, either underweight (body mass index $<18.5$ ) or obese (body mass index $\geq 30 ; P=0.0379$ ). There was no difference in the rate of classic heterotaxy by sex of cases as compared with controls ( 46.8 vs. $51.0 \%$ male, respectively; $P=0.4930$ ). Infants with classic heterotaxy were born at an earlier gestational age, with a mean 
Table 1 Selected characteristics of cases with classic heterotaxy and a random sample of New York State (NYS) live births

\begin{tabular}{|c|c|c|c|}
\hline Characteristic & $\begin{array}{l}\text { NYS births } \\
(n=7,683)\end{array}$ & $\begin{array}{c}\text { Heterotaxy } \\
\text { cases }(n=77)\end{array}$ & $P$ value \\
\hline \multicolumn{3}{|l|}{ Maternal age (years) } & $1.0000^{a}$ \\
\hline$<20$ & $612(7.97)$ & $6(7.79)$ & \\
\hline $20-34$ & $5,637(73.37)$ & $57(74.03)$ & \\
\hline$\geq 35$ & $1,434(18.66)$ & $14(18.18)$ & \\
\hline \multicolumn{3}{|c|}{ Maternal race/ethnicity } & $0.0108^{a}$ \\
\hline Non-Hispanic white & $4,339(56.59)$ & $33(42.86)$ & \\
\hline African American & $1,369(17.86)$ & $11(14.29)$ & \\
\hline Hispanic & $1,359(17.73)$ & $21(27.27)$ & $0.0356^{a, b}$ \\
\hline Asian & $520(6.78)$ & $11(14.29)$ & $0.0194^{\mathrm{a}, \mathrm{c}}$ \\
\hline Other & $80(1.04)$ & $1(1.30)$ & \\
\hline \multicolumn{3}{|c|}{ Maternal education (years) } & $0.0027^{\circ}$ \\
\hline$<12$ & $1,335(17.56)$ & $23(30.26)$ & \\
\hline 12 & $2,221(29.22)$ & $26(34.21)$ & \\
\hline$>12$ & $4,045(53.22)$ & $27(35.53)$ & \\
\hline \multicolumn{3}{|l|}{ Parity, $n$ (\%) } & $0.8163^{\circ}$ \\
\hline Nulliparous & $3,165(41.19)$ & $33(42.86)$ & \\
\hline Multiparous & $4,518(58.81)$ & $44(57.14)$ & \\
\hline \multicolumn{3}{|l|}{ Maternal smoking } & $1.0000^{\circ}$ \\
\hline No & $6,911(90.09)$ & $70(90.91)$ & \\
\hline Yes & $760(9.91)$ & $7(9.09)$ & \\
\hline $\begin{array}{l}\text { Prepregnancy } \\
\text { maternal BMI }\left(\mathrm{kg} / \mathrm{m}^{2}\right)\end{array}$ & $(n=4,197)^{d}$ & $(n=39)^{d}$ & $0.0379^{a}$ \\
\hline$<18.5$ & $171(4.07)$ & $4(10.26)$ & \\
\hline $18.5-24.9$ & $2,197(52.35)$ & $20(51.28)$ & \\
\hline $25-29.9$ & $992(23.64)$ & $4(10.26)$ & \\
\hline$\geq 30$ & $837(19.94)$ & $11(28.21)$ & \\
\hline \multicolumn{3}{|l|}{ Infant sex } & $0.4930^{a}$ \\
\hline Male & $3,916(50.97)$ & $36(46.75)$ & \\
\hline Female & 3,767 (49.03) & $41(53.25)$ & \\
\hline $\begin{array}{l}\text { Infant gestational age } \\
\text { (mean days } \pm \text { SD) }\end{array}$ & $275.4 \pm 16.8$ & $269.8 \pm 21.2$ & $0.0316^{e}$ \\
\hline $\begin{array}{l}\text { Infant birth weight } \\
\text { (mean grams } \pm \text { SD) }\end{array}$ & $3,330.2 \pm 577.0$ & $2,970.4 \pm 742.5$ & $<0.0001^{\mathrm{e}}$ \\
\hline
\end{tabular}

Data are $n(\%)$ unless otherwise indicated.

a $P$ value determined using Fisher's exact test. ${ }^{b} P$ value assigned independently to Hispanic versus all other races. ${ }^{c} P$ value assigned independently to Asian versus all other races. ${ }^{d}$ Demographic variables are not available for all controls and are noted where a substantial proportion of data are missing. ${ }^{e} P$ value determined using $t$-test. BMI, body mass index.

of 269 days $\left(38^{+} 3 / 7\right.$ weeks) for cases versus 275 days $\left(39^{+} 2 / 7\right.$ weeks) for controls $(P=0.0316)$, and, similarly, had lower birth weights (mean of 2,970 vs. $3,330 \mathrm{~g} ; P \leq 0.0001$ ). When adjusted for gestational age using Fenton infantile growth charts, the mean birth weights were in the 33rd percentile in cases and the 48th percentile in controls. Characterization of cases of classic heterotaxy by phenotype revealed that $32 \%$ were associated with asplenia $(n=25), 14 \%$ had polysplenia $(n=11)$, and $54 \%$ had undefined splenic status $(n=41)$. The most frequently reported cardiac malformations were single ventricle defects $(n$ $=23)$, double outlet right ventricle $(n=21)$, dextro-transposition of the great arteries $(n=19)$, pulmonic stenosis $(n=12)$, atrioventricular septal defect $(n=10)$, total anomalous pulmonary venous return $(n=8)$, and pulmonic atresia $(n=5)$.

We genotyped 74 samples with classic heterotaxy, which resulted in 5,794 PennCNV calls in the primary microarray analysis. After applying exclusionary filters outlined in the Materials and Methods, 20 CNVs in 19 different cases (Table 2) were prioritized and selected for follow-up based on minimal overlap with published CNVs in DGVa/DGV2 and/or recurrence in more than one subject. These included six genic heterozygous deletions and four genic duplications (in six cases); two heterozygous deletions and five duplications intersecting at least one GENCODE transcript; and three intergenic heterozygous deletions. The CNVs ranged in size from $20 \mathrm{~kb}$ to $8.4 \mathrm{Mb}$. $\mathrm{CNV}$ s identified only in the secondary analysis or by the cnvPartition algorithm alone were not prioritized for follow-up studies. All 20 candidate CNVs were validated using quantitative polymerase chain reaction with specific amplicons. Sequencing revealed no known pathologic heterotaxy mutations involving CFC1, ZIC3, FOXH1, ACVR2B, or NODAL in the 19 subjects carrying a candidate $\mathrm{CNV}$; other variants detected are listed in Supplementary Table S4 online).

In individual cases, we identified rare CNVs involving several genes from the transforming growth factor- $\beta$ superfamily, including a $4.2-\mathrm{Mb}$ deletion at $20 \mathrm{p} 12.3$ involving the coding region of bone morphogenic protein 2 (BMP2) (Figure 1), a 224-kb deletion at 3q28 involving the fibroblast growth factor 12 (FGF12) gene, and an 824-kb duplication of 2p24.1, which includes the GDF7 gene encoding growth differentiation factor 7. These are rare variants and were not observed in our control sample $(n=138)$ or reported in the DGV. The phenotypes of these subjects included situs inversus with single ventricle and interrupted aortic arch; situs inversus with asplenia, single ventricle, right aortic arch, and total anomalous pulmonary venous return; and situs inversus with duodenal atresia and ventricular septal defect, respectively.

We also identified two heterozygous deletions that overlapped with CNVs previously reported in a heterotaxy cohort. ${ }^{20}$ The first was a $167-\mathrm{kb}$ deletion at 1q23.1 in the coding region for the myeloid cell nuclear differentiation antigen $(M N D A)$ gene (Figure 2). The case's phenotype was characterized by polysplenia, interrupted inferior vena cava, and persistent left superior vena cava. The second variant was a large $8.4-\mathrm{Mb}$ terminal deletion beginning at 10q26.13 and involving approximately 50 genes (Figure 3). The case was characterized by asplenia, double outlet right ventricle, total anomalous pulmonary vascular return, and hypoplastic left heart syndrome. No additional clinical information was available for this patient.

A similar $\sim 20-\mathrm{kb}$ duplication at $4 \mathrm{q} 25$ was discovered in three separate cases (IDs 5-7; Table 2). All three duplications overlapped one or more exons of the oligosaccharyltransferase complex subunit (OSTC) gene (Supplementary Figure S1 online). There was no overlap with any variant from the DGV, and it 
Table 2 Rare CNVs identified in cases with classic heterotaxy phenotype

\begin{tabular}{|c|c|c|c|c|c|c|}
\hline Locus & Genomic coordinates & Size (bp) & Type & $\begin{array}{c}\text { Case } \\
\text { ID }\end{array}$ & Race & Gene(s)/transcript(s) \\
\hline 1q23.1 & Chr1: $158,681,591-158,848,721$ & 167,131 & Het Del & 1 & Hispanic & $\begin{array}{l}\text { MNDA, OR6K3, OR6K6, OR6N1, } \\
\text { OR6N2 }\end{array}$ \\
\hline $2 \mathrm{p} 24.1$ & $\begin{array}{l}\text { (Chr2: 20,771,563-21,595,962) } \\
\text { Chr2: 20,771,563-21,146,521 } \\
\text { Chr2: 21,186,891-21,595,962 }\end{array}$ & $\begin{array}{c}(824,400)^{b} \\
374,959 \\
409,072\end{array}$ & Dupl & 2 & White & $\begin{array}{l}\text { GDF7, HS1BP3, c2orf43, APOB, RP11- } \\
\text { 130L8.2, HS1BP3-IT1, AC012065.7, } \\
\text { RP11-130L8.1, AC012361.1, RP11- } \\
\text { 116D2.1, TDRD15, AC067959.1 }\end{array}$ \\
\hline $3 p 26.1$ & Chr3: 4,420,255-4,805,536 & 385,282 & Het Del & 3 & White & SUMF1, ITPR1, ITPR1-AS1, EGOT \\
\hline $3 q 28$ & Chr3: 192,014,722-192,238,859 & 224,138 & Het Del & 4 & Hispanic & FGF12 \\
\hline \multirow[t]{3}{*}{$4 q 25$} & Chr4: 109,552,090-109,573,496 & 21,407 & Dupl & 7 & Hispanic & OSTC, RNU6-431P \\
\hline & Chr4: 109,553,331-109,573,496 & 20,166 & Dupl & 6 & Hispanic & OSTC, RNU6-431P \\
\hline & Chr4: 109,555,691-109,577,756 & 22,066 & Dupl & 5 & Hispanic & OSTC, RNU6-431P \\
\hline $5 q 34$ & Chr5: 162,646,452-162,672,927 & 26,476 & Dupl & 16 & Other & RP11-541P9.3 \\
\hline $\begin{array}{l}7 q 11.21- \\
7 q 11.22\end{array}$ & Chr7: $66,773,745-67,077,894^{\mathrm{b}, \mathrm{c}}$ & $304,150^{b, c}$ & Dupl & 17 & Asian & $\begin{array}{l}\text { RP11-16604.5, STAG3L4, } \\
\text { RP11-16604.6, AC006480.1 }\end{array}$ \\
\hline $7 q 11.22$ & Chr7: $67,137,234-67,530,357^{\mathrm{b}, \mathrm{c}}$ & $393,124^{b, c}$ & Dupl & 17 & Asian & RP4-736H5.3, RP11-358M3.1 \\
\hline $9 p 23$ & Chr9: $13,656,508-13,851,118$ & 194,611 & Het Del & 18 & White & Intergenic \\
\hline \multirow{3}{*}{$\begin{array}{l}\text { 10q26.13- } \\
\text { telomere }\end{array}$} & $($ Chr10: 127,037,255-135,477,883) & $(8,440,629)^{\mathrm{b}}$ & \multirow{3}{*}{$\begin{array}{l}\text { Het } \\
\text { Del }\end{array}$} & \multirow[t]{3}{*}{9} & \multirow[t]{3}{*}{ Hispanic } & \multirow{3}{*}{$\begin{array}{l}\text { Terminal deletion, }>50 \text { genes, } \\
\text { FOXI2, NKX6-2 }\end{array}$} \\
\hline & Chr10: 127,037,255-133,637,385 & $6,600,131$ & & & & \\
\hline & Chr10: $133,643,936-135,477,883$ & $1,833,948$ & & & & \\
\hline $11 q 22.3$ & Chr11: $109,665,058-109,803,414$ & 138,357 & Dupl & 19 & Black & $R P 11-262 A 12.1$ \\
\hline $13 q 21.31$ & Chr13: 62,866,393-63,004,983 & 138,591 & Dupl & 5 & Hispanic & LINC00459, LINC01074 \\
\hline $22 q 13.2$ & Chr22: 42,303,839-42,442,157 & 138,319 & Dupl & 12 & White & $\begin{array}{l}\text { SHISA8, TNFRSF13C, CENPM, } \\
\text { BK250D10, SEPT3, WBP2NL, MIR378I, } \\
\text { CTA-250D10.23, LINC00634, CTA- } \\
\text { 250D10.19 }\end{array}$ \\
\hline
\end{tabular}

Bolded items are highlighted in the figures and/or Discussion section.

anless otherwise specified, coordinates (hg19) predicted using PennCNV (primary analysis). ${ }^{\circ}$ Coordinates in parentheses are predicted based on evidence from PennCNV,

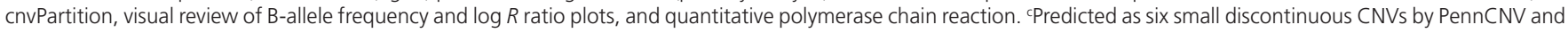
predicted as two small discontinuous CNVs by cnvPartition. Quantitative polymerase chain reaction analysis demonstrated at least two CNVs in this area.

CNV, copy-number variant; Dupl, duplication; Het Del, heterozygous deletion.

was absent in the quality control samples $(n=6)$. All three cases involved Hispanic mothers. Two cases were characterized by double outlet ventricle and situs inversus abdominis and a third case with dextrocardia, situs inversus abdominis, stenosis of the bowel, and imperforate anus. Among 138 control subjects screened in a follow-up analysis, one carried a similar duplication (all four copy-number assays showed a duplication across the OSTC gene region). The mother of the control subject also was Hispanic.

\section{DISCUSSION}

It is well established that left-to-right asymmetry depends on the unilateral expression of NODAL within the developing embryo. Several members of the transforming growth factor- $\beta$ superfamily involved in the nodal signaling pathway, including NODAL, LEFTY2, and GDF1, have previously been implicated in human heterotaxy. We present several rare CNVs involving candidate genes from the transforming growth factor- $\beta$ superfamily that have not previously been reported in human 

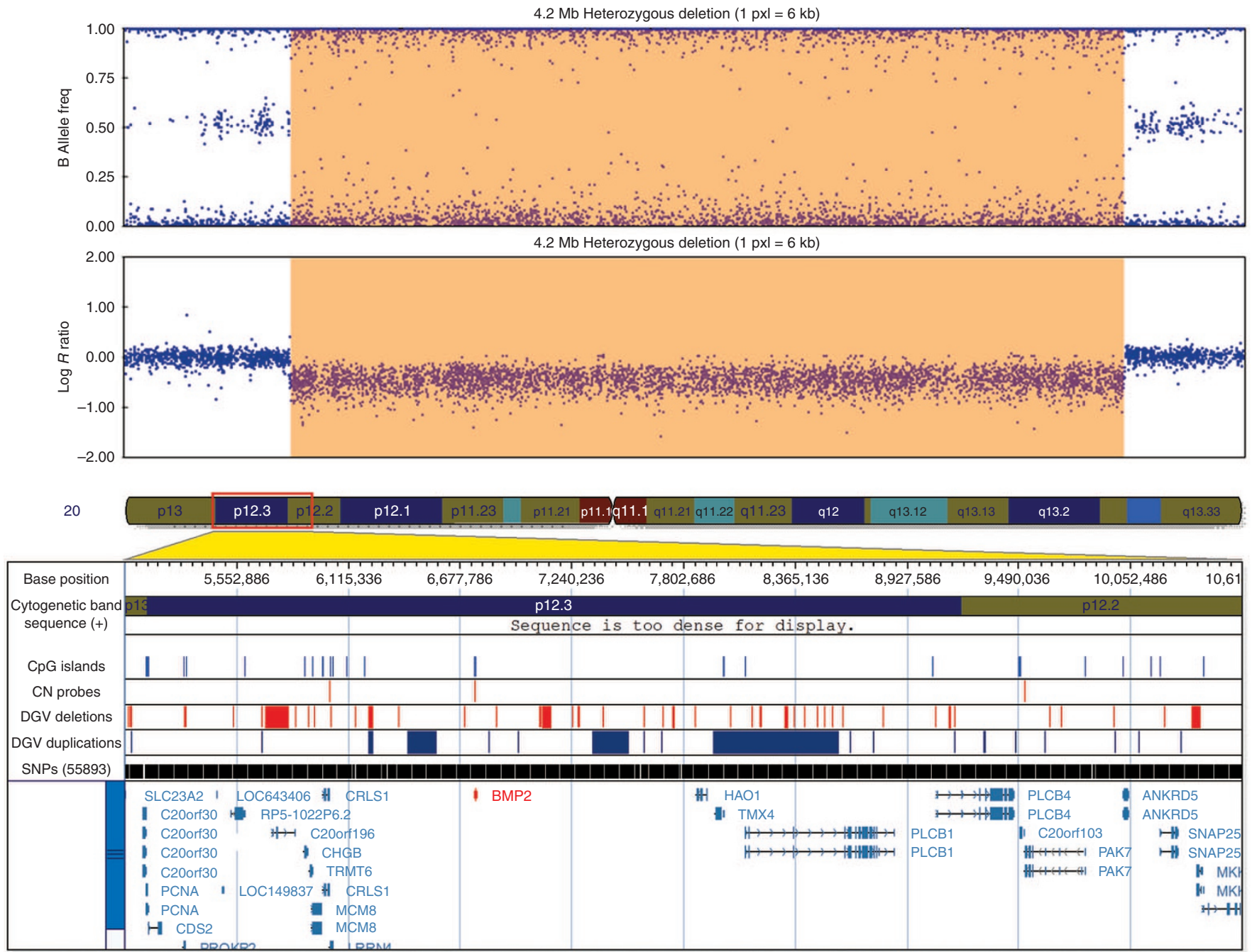

Figure 1 Visualization of the 4.2-Mb heterozygous deletion in case 11 encompassing BMP2 (chromosome 20p), exported from the Illumina Genome Viewer. The top panel depicts B-allele frequency (ratio of minor to major alleles), and the bottom panel depicts the log $R$ ratio data (signal intensity). Tracks provided by Illumina show cytobands, CpG islands, and the location of single-nucleotide polymorphisms on the array. Custom tracks were created to display the location targeted by copy-number assays used to validate copy-number variants (CNVs; "CN probes"), and copy-number losses ("DGV Deletions," shown in red) and gains ("DGV Duplications," shown in blue), both of which were downloaded from the DGVa database. Genes/transcripts overlapping or intersecting CNVs and genes immediately flanking the CNVs are listed below the panels. Genes with potential relevance to heterotaxy are highlighted in red. CNV calls made using the PennCNV algorithm are highlighted (heterozygous deletions in orange and duplications in blue). hg19 Coordinates are shown.

heterotaxy, including a deletion encompassing $B M P 2$, a duplication encompassing GDF7, and a deletion in FGF12.

Bone morphogenic proteins (bmps) are expressed in a variety of tissues including the node and lateral plate mesoderm of the early developing embryo and, later, in bone, cartilage, and the heart. Animal models have shown that bmps affect left-to-right patterning, serving as either inhibitors or activators of nodal signaling depending on where in the embryo they are expressed. ${ }^{29}$ For example, increased bmp signaling on the right side of the embryo results in situs abnormalities, whereas increased signaling on the left seems to have no phenotypic effect. ${ }^{30}$ In zebrafish, abnormalities of bmp2/bmp4 signaling result in laterality defects of the heart. ${ }^{31}$ A Bmp2 knockout in mice has recently been shown to result in impaired ventral folding in the developing embryo. Affected embryos displayed various defects including an abnormal head-to-heart relationship, failure of cardiac looping, and lack of foregut invagination. ${ }^{32}$ While animal studies support a link between bmp2/bmp4 mutations and laterality disorders, we are the first to report a $B M P 2$ deletion in association with a human heterotaxy phenotype.

In animals, $G d f 7$, otherwise known as $B m p 12$, is also involved in bmp signaling and may play a role in NODAL expression. In mice, $G d f 7$ functions as an inductive signal from the embryonic roof plate required for neuronal patterning in the dorsal spinal cord. In zebrafish, morpholino knockdown of $g d f 7$ was not associated with defects in laterality or other defects. ${ }^{20}$ In mouse models, however, mutations in Gdf7 were associated with other disease phenotypes, including hydrocephalus and reproductive anomalies. ${ }^{20,33}$

The function of FGF12 is not fully understood in humans; in animals, however, the $f g f$ family possesses broad mitogenic 

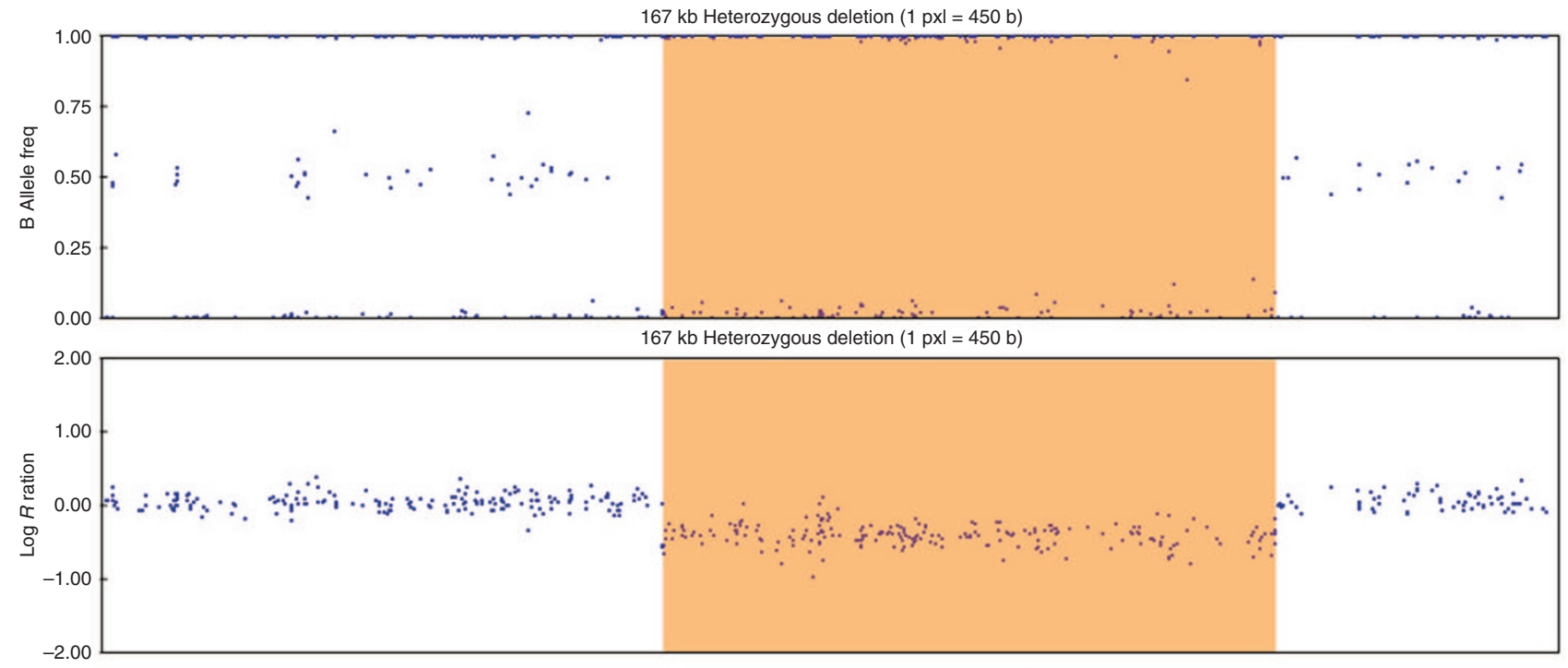

1

p31.3 p31.1

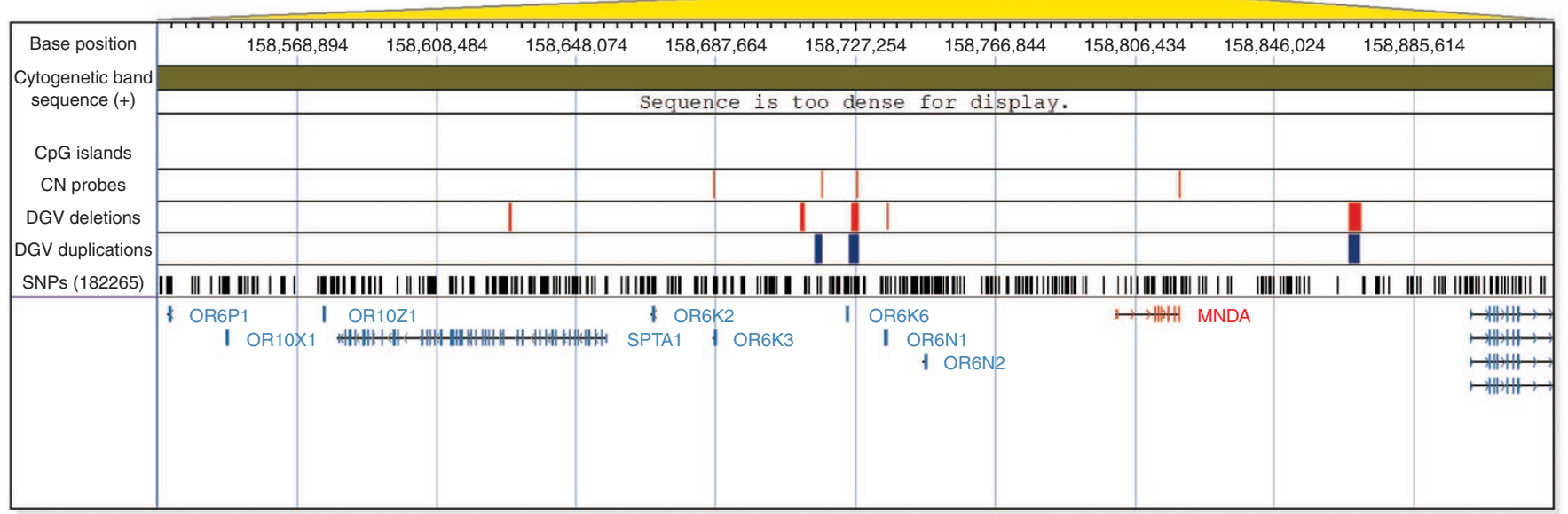

Figure 2 Visualization of the 167-kb heterozygous deletion in case 1 encompassing the MNDA gene on chromosome 1q, exported from the Illumina Genome Viewer. The top panel depicts B-allele frequency (ratio of minor to major alleles), and the bottom panel depicts the log $R$ ratio data (signal intensity). Tracks provided by Illumina show cytobands, CpG islands, and the location of single-nucleotide polymorphisms on the array. Custom tracks were created to display the location targeted by copy-number assays used to validate copy-number variants (CNVs; "CN probes"), and copy-number losses ("DGV deletions," shown in red) and gains ("DGV duplications," shown in blue), both of which were downloaded from the DGVa database. Genes/transcripts overlapping or intersecting CNVs and genes immediately flanking the CNVs are listed below the panels. Genes with potential relevance to heterotaxy are highlighted in red. CNV calls made using the PennCNV algorithm are highlighted (heterozygous deletions in orange and duplications in blue). hg19 Coordinates are shown.

activities and is involved in a variety of biological processes, including embryonic development, cell growth, morphogenesis, and tissue repair. ${ }^{34}$ In zebrafish, $f g f$ signaling regulates nodal-dependent endoderm induction during gastrulation, one of the earliest steps in the establishment of asymmetry. ${ }^{35} \mathrm{In}$ addition, impaired $f g f$ signaling causes defective cilia formation in Kupffer's vesicle, the laterality organ in zebrafish. ${ }^{36}$

Interestingly, we are the second group to report a large terminal deletion beginning at 10q26.13 and a deletion involving the MNDA gene at 1q23.1 associated with heterotaxy. In 2011, Fakhro et al..$^{20}$ published similar variants identified in a sample of 262 patients with various anomalies of situs, including isolated CHD. The deletion at 10q26.13 reported here is smaller
(Chr10:127,037,255-135,477,883; hg19) than the deletion published by Fakhro et al. ${ }^{20}$ (Chr10:125,445,010-135,524,010; coordinates lifted over from hg18 to hg19), which narrows the number of gene candidates from approximately 300 to 50 genes. This region is of particular interest because it is located downstream from NODAL and contains the coding regions of several transcription factors including FOXI2 and NKX6-2; members of both these gene families have been implicated in left-to-right specification. Members of the Fox gene family are essential for early development and differentiation. In humans, FOXH1 is a key transcription factor in the nodal signaling pathway. ${ }^{37}$ In a recent study, foxi2-depleted Xenopus blastulas were noted to have delayed gastrulation movements with subsequent major 

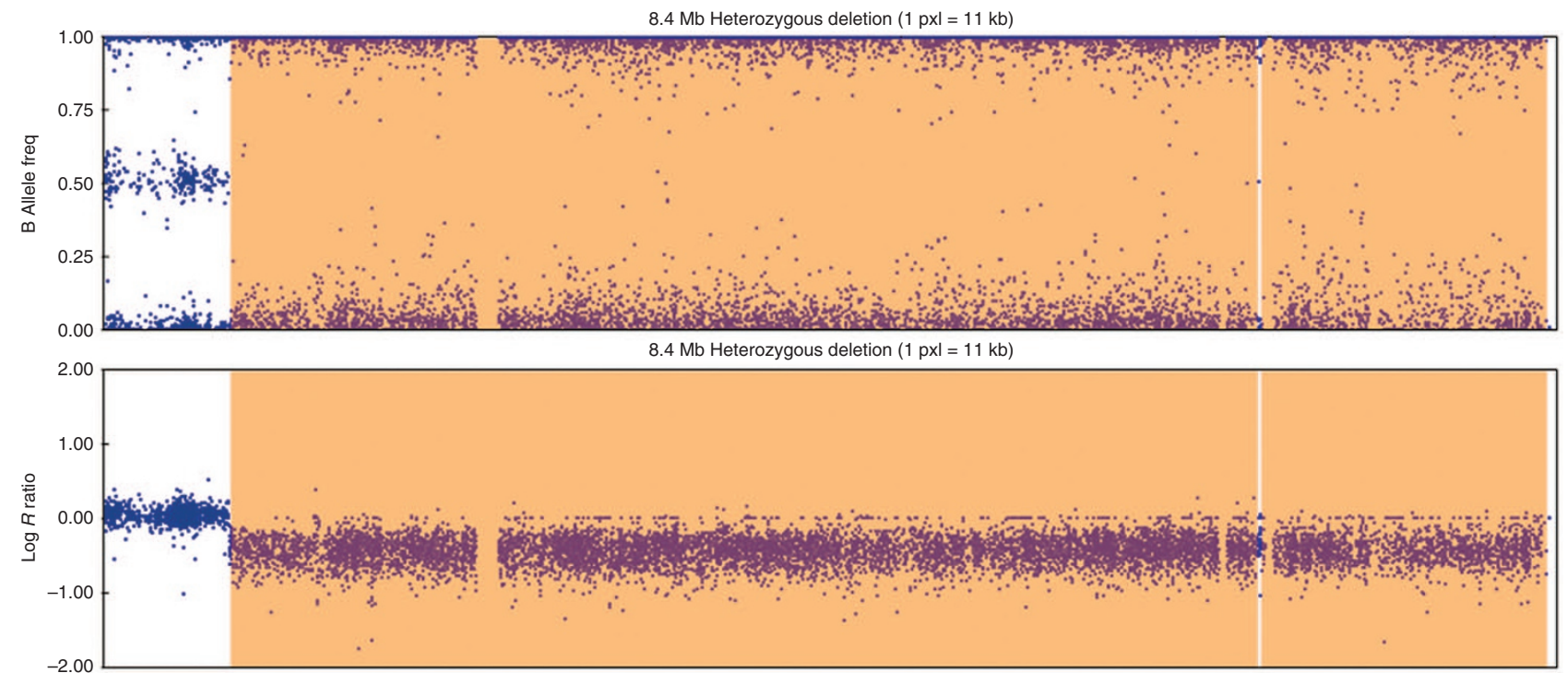

\begin{tabular}{|l|l|l|l|l|l|l|l|l|l|l|l|l|l|}
\hline 10 & $\mathrm{p} 14$ & $\mathrm{p} 13$ & $\mathrm{p} 12.1$ & $\mathrm{q} 21.1$ & $\mathrm{q} 21.3$ & $\mathrm{q} 22.1$ & $\mathrm{q} 22.3 \mathrm{q} 23.1$ & & $\mathrm{q} 25.1$ & $\mathrm{q} 25.3$ \\
\hline
\end{tabular}

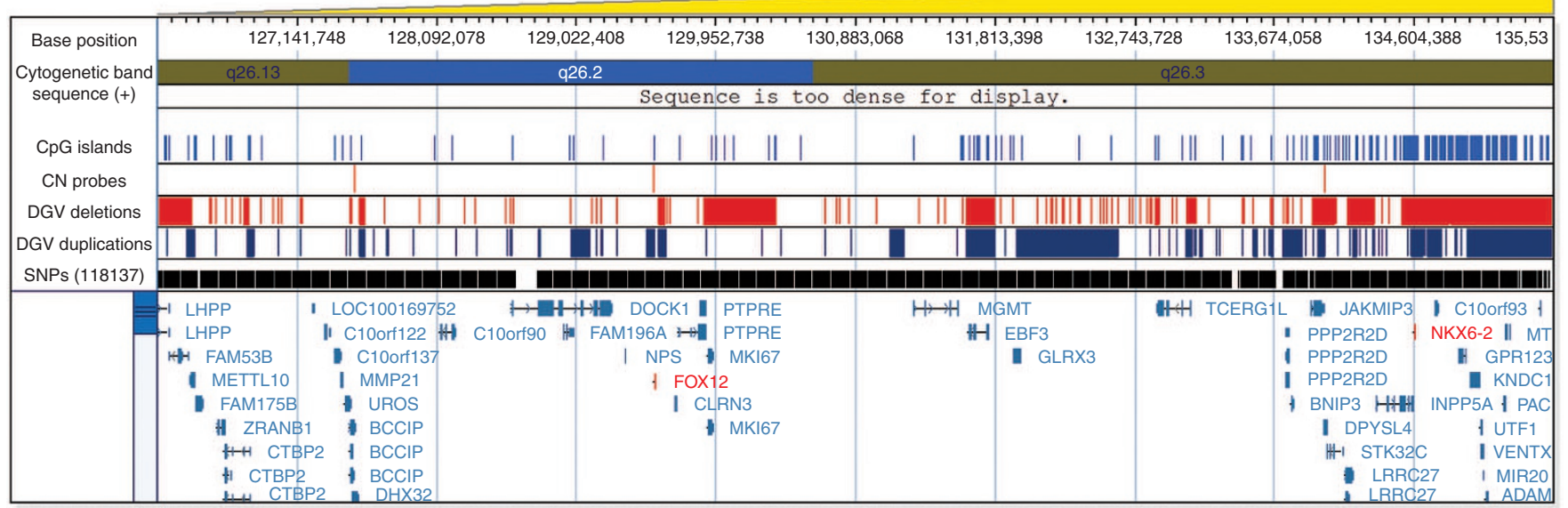

Figure 3 Visualization of the 8.4-Mb heterozygous deletion in case 9 including FOXI2 and NKX6-2 (chromosome 10q), exported from the Illumina Genome Viewer. The top panel depicts B-allele frequency (ratio of minor to major alleles), and the bottom panel depicts the log $R$ ratio data (signal intensity). Tracks provided by Illumina show cytobands, CpG islands, and the location of single-nucleotide polymorphisms on the array. Custom tracks were created to display the location targeted by copy-number assays used to validate copy-number variants (CNVs; "CN probes"), and copy-number losses ("DGV deletions," shown in red) and gains ("DGV duplications," shown in blue), both of which were downloaded from the DGVa database. A subset of genes/transcripts overlapping or intersecting CNVs and genes immediately flanking the CNVs are listed below the panels. Genes with potential relevance to heterotaxy are highlighted in red. CNV calls made using the PennCNV algorithm are highlighted (heterozygous deletions in orange and duplications in blue). hg19 Coordinates are shown. Not all genes included in the deletion are listed.

patterning abnormalities in the embryos. ${ }^{38}$ Mutations involving the NK homeobox protein family have already been associated with heterotaxy, specifically NKX2-5 mutations. ${ }^{16}$ Animal studies involving $n k \times 6-2$ suggest that it plays a role in neuronal differentiation and axis formation within the central nervous system. ${ }^{39}$

Fakhro et al. ${ }^{20}$ also reported a $102-\mathrm{kb}$ deletion at $1 \mathrm{q} 23.1$ (Chr1:158,746,376-158,848,376; hg19), whereas we discovered a larger 167-kb deletion in the same region (Chr1:158,681,591158,848,721; hg19). Both deletions overlap the MNDA gene and the olfactory receptor $6 \mathrm{~N} 2$ gene (OR6N2). The MNDA gene is expressed in the nuclei of granulocytes and is thought to play a role in blood cell responses to interferon and apoptosis.
Additional gene functions may exist, but animal studies are lacking. Olfactory receptor genes are not known to be related to heterotaxy.

Despite our prediction that restricting our case definition to classic heterotaxy would result in greater genetic homogeneity, we found only one recurring variant that met inclusion criteria. The variant of interest, a microduplication at $4 \mathrm{q} 25$, is located upstream from PITX2, a known regulator of nodal signaling, and codes for the OSTC gene. The OSTC gene is involved in $\mathrm{N}$-linked protein glycosylation, a common post-translational modification of cell membrane proteins. This is noteworthy because disorders of glycosylation have recently been suggested to play a role in laterality defects. ${ }^{20}$ For example, CRELD1 gene 
mutations alter an amino acid at the $\mathrm{N}$-linked glycosylation site, resulting in impaired glycosylation and abnormal protein folding. ${ }^{15}$ Follow-up screening in 138 unaffected controls resulted in the identification of a similar duplication in one control subject. All three heterotaxy cases and the one control subject with the $4 \mathrm{q} 25$ duplication were noted to have Hispanic mothers. This variant is unlikely to be associated with heterotaxy specifically, but it may represent a rare CNV within the Hispanic population that has not previously been reported.

This study has several strengths. To our knowledge, this is the first microarray analysis of cases involving a restricted definition of heterotaxy. Unlike previous studies, we did not include isolated splenic anomalies, isolated cardiovascular malformations, or isolated situs inversus totalis to explore whether genetic homogeneity existed among more complex cases of heterotaxy. In addition, we excluded any subjects with major non-heterotaxy-related birth defects that could otherwise be the cause of rare CNVs detected in our analysis. In addition, we investigated population characteristics associated with classic heterotaxy and are the first to note an increased incidence of classic heterotaxy among Hispanic infants, in addition to Asian infants, suggesting that genetic variants play a role in heterotaxy. We also noted lower birth weights among study infants, even after controlling for earlier gestational age at birth. The significance of this finding is unclear, although growth abnormalities often are associated with genetic syndromes, further supporting that genetic factors play a role in heterotaxy.

DBSs often are used for targeted genetic analysis. Recent studies have shown that genome-wide single-nucleotide polymorphism genotypes can be reliably obtained from wholegenome amplified DNA extracted from archived DBSs. To the best of our knowledge, ours is the first study to provide data demonstrating that CNVs can also reliably be detected from DNA extracted from DBSs and without prior whole-genome amplification. DBSs are routinely collected from infants born in the United States for routine metabolic screening. Use of DBSs for microarray testing may serve useful in clinical practice, particularly in circumstances in which genetic testing would normally be precluded, such as immediately following a blood transfusion, after an unexpected death, or when traditional venipuncture would be difficult to perform.

The primary goal of our study was to identify CNVs unique to classic heterotaxy, and we also report population characteristics of classic heterotaxy. Although we are unique in being able to identify cases from such a large and diverse population as that found in NYS, selecting and characterizing cases based on diagnostic codes in a population-based registry can have certain limitations. For example, estimating the prevalence of malformations from live births can be affected by early losses or terminations that may not have been captured completely by the registry. In addition, we may have missed or excluded cases that were incorrectly coded or not reported, although this is uncommon. Furthermore, classifying heterotaxy phenotypes based on registry codes is less reliable than a clinic-based study, in which laterality defects can be systematically characterized by the investigating physicians. Heterotaxy cases would typically be verified by imaging or autopsy; with registry data, however, we have to presume that diagnoses were confirmed and coded appropriately. From a genomic standpoint, assessing the clinical relevance of CNVs can be problematic given the potential inaccuracies in mapping breakpoints, estimating the frequency of CNVs, and considering the potential for bias in population-based genomic databases such as $\mathrm{DGV}^{40} \mathrm{We}$ also may have been too restrictive by including for follow-up CNVs that had only minimal overlap with variants published in the DGV. Ideally, regions of interest should be followed up with translational studies; however, it can be difficult to correlate animal models with human disease. For instance, GDF7 is a strong candidate based on biologic plausibility, but animal studies do not support its role in laterality disorders.

In conclusion, although we hypothesized that classic heterotaxy was more likely to be associated with genetic homogeneity, our results did not support a specific phenotype-genotype link. We did, however, discover several novel CNVs involving genes with known roles in left-to-right specification that have not previously been implicated in human heterotaxy, including $B M P 2, G D F 7$, and FGF12. In addition, we identified rare CNVs reported previously in heterotaxy cases, including a terminal deletion at 10q26.13 and a microdeletion of the MNDA gene at 1q23.1. The potential role of these genes and regions in the etiology of classic heterotaxy in humans should be investigated further. Furthermore, we demonstrated that DBS DNA can reliably be used for $\mathrm{CNV}$ analysis.

\section{SUPPLEMENTARY MATERIAL}

Supplementary material is linked to the online version of the paper at http://www.nature.com/gim

\section{ACKNOWLEDGMENTS}

This work was funded by the Intramural Research Program of the National Institutes of Health, Eunice Kennedy Shriver National Institute of Child Health and Human Development (contracts HHSN275201100001I and HHSN27500005; NICHD contract N01-DK-73431) and the National Human Genome Research Institute. Funding for the DECIPHER project was provided by the Wellcome Trust. We thank Michael Tsai and Natalie Weir at the Minnesota Core Laboratories and the staff at the Biomedical Genomics Center Facility at the University of Minnesota for microarray genotyping; Emily C. McGrath, Adam C. Gearhart, April J. Atkins, Deborah K. West, and Zoe Edmunds at the Wadsworth Center, New York State Department of Health, for Sanger sequencing and laboratory and technical assistance; Sandra D. Richardson at the Congenital Malformations Registry, New York State Department of Health, for data management; Matthew Shudt and Zhen Zhang at the Wadsworth Center Applied Genomic Technologies Core for next-generation sequencing; and Nathan Pankratz, University of Minnesota, and Karl G. Hill, Social Development Research Group, University of Washington, for generously sharing population B-allele frequency and GC content files for PennCNV software. This study makes use of data generated by 
the DECIPHER Consortium. A full list of centers that contributed to the generation of the data is available from http://decipher. sanger.ac.uk and via email from decipher@sanger.ac.uk. Those who carried out the original analysis and collection of the data bear no responsibility for the further analysis or interpretation. Some data used for comparison in this article were obtained from the International Standards for Cytogenomic Arrays Consortium database (http://www.iscaconsortium.org), which generates this information using the National Center for Biotechnology Information's database of genomic structural variation (dbVar, http:// www.ncbi.nlm.nih.gov/dbvar/), study nstd37. Samples and associated phenotype data were provided by International Standards for Cytogenomic Arrays Consortium member laboratories.

\section{DISCLOSURE}

The authors declare no conflict of interest.

\section{REFERENCES}

1. Jacobs JP, Anderson RH, Weinberg PM, et al. The nomenclature, definition and classification of cardiac structures in the setting of heterotaxy. Cardiol Young 2007;17(suppl 2):1-28.

2. Zhu L, Belmont JW, Ware SM. Genetics of human heterotaxias. Eur J Hum Genet 2006; 14:17-25.

3. Lin AE, Ticho BS, Houde K, Westgate MN, Holmes LB. Heterotaxy: associated conditions and hospital-based prevalence in newborns. Genet Med 2000;2:157-172.

4. Ware SM, Peng J, Zhu L, et al. Identification and functional analysis of ZIC3 mutations in heterotaxy and related congenital heart defects. Am J Hum Genet 2004;74:93-105.

5. Ferenz C, Loffredo CA, Correa-Villaseñor A. Genetic and environmental risk factors of major cardiovascular malformations. The Baltimore-Washington Infant Study 1981-1989, Perspectives in Pediatric Cardiology, Volv. Armonk, NY: Futura Publishing, 1997.

6. Kim SJ, Kim WH, Lim HG, Lee JY. Outcome of 200 patients after an extracardiac Fontan procedure. J Thorac Cardiovasc Surg 2008;136:108-116.

7. Correa-Villaseñor A, McCarter R, Downing J, Ferencz C. White-black differences in cardiovascular malformations in infancy and socioeconomic factors. The Baltimore-Washington Infant Study Group. Am J Epidemiol 1991;134:393402.

8. Nakhleh N, Francis R, Giese RA, et al. High prevalence of respiratory ciliary dysfunction in congenital heart disease patients with heterotaxy. Circulation 2012;125:2232-2242.

9. Swisher M, Jonas R, Tian X, Lee ES, Lo CW, Leatherbury L. Increased postoperative and respiratory complications in patients with congenital heart disease associated with heterotaxy. J Thorac Cardiovasc Surg 2011;141:637644, 644 e631-633.

10. Bamford RN, Roessler E, Burdine RD, et al. Loss-of-function mutations in the EGF-CFC gene CFC1 are associated with human left-right laterality defects. Nat Genet 2000;26:365-369.

11. Mohapatra B, Casey B, Li H, et al. Identification and functional characterization of NODAL rare variants in heterotaxy and isolated cardiovascular malformations. Hum Mol Genet 2009;18:861-871.

12. Roessler E, Ouspenskaia MV, Karkera JD, et al. Reduced NODAL signaling strength via mutation of several pathway members including FOXH1 is linked to human heart defects and holoprosencephaly. Am J Hum Genet 2008;83:18-29.

13. Kosaki R, Gebbia M, Kosaki K, et al. Left-right axis malformations associated with mutations in ACVR2B, the gene for human activin receptor type IIB. Am J Med Genet 1999;82:70-76.

14. Robinson SW, Morris CD, Goldmuntz E, et al. Missense mutations in CRELD1 are associated with cardiac atrioventricular septal defects. Am J Hum Genet 2003;72:1047-1052.
15. Zhian S, Belmont J, Maslen CL. Specific association of missense mutations in CRELD1 with cardiac atrioventricular septal defects in heterotaxy syndrome. Am J Med Genet A 2012;158A:2047-2049.

16. Watanabe Y, Benson DW, Yano S, Akagi T, Yoshino M, Murray JC. Two novel frameshift mutations in NKX2.5 result in novel features including visceral inversus and sinus venosus type ASD. J Med Genet 2002;39:807-811.

17. Kennedy MP, Omran H, Leigh MW, et al. Congenital heart disease and other heterotaxic defects in a large cohort of patients with primary ciliary dyskinesia. Circulation 2007:115:2814-2821.

18. Gebbia M, Ferrero GB, Pilia G, et al. X-linked situs abnormalities result from mutations in ZIC3. Nat Genet 1997:17:305-308.

19. Sutherland MJ, Ware SM. Disorders of left-right asymmetry: heterotaxy and situs inversus. Am J Med Genet C Semin Med Genet 2009;151C:307-317.

20. Fakhro KA, Choi M, Ware SM, et al. Rare copy number variations in congenital heart disease patients identify unique genes in left-right patterning. Proc Natl Acad Sci USA 2011;108:2915-2920.

21. De Luca A, Sarkozy A, Consoli F, et al. Familial transposition of the great arteries caused by multiple mutations in laterality genes. Heart 2010;96:673-677.

22. Saavedra-Matiz CA, Isabelle JT, Biski CK, et al. Cost-effective and scalable DNA extraction method from dried blood spots. Clin Chem 2013;59:1045-1051.

23. Wang K, Li M, Hadley D, et al. PennCNV: an integrated hidden Markov model designed for high-resolution copy number variation detection in whole-genome SNP genotyping data. Genome Res 2007;17:1665-1674.

24. Iafrate AJ, Feuk L, Rivera MN, et al. Detection of large-scale variation in the human genome. Nat Genet 2004;36:949-951.

25. Harrow J, Frankish A, Gonzalez JM, et al. GENCODE: the reference human genome annotation for The ENCODE Project. Genome Res 2012;22:17601774

26. Pruitt KD, Harrow J, Harte RA, et al. The consensus coding sequence (CCDS) project: identifying a common protein-coding gene set for the human and mouse genomes. Genome Res 2009;19:1316-1323.

27. Wessels MW, Willems PJ. Genetic factors in non-syndromic congenital heart malformations. Clin Genet 2010;78:103-123.

28. Vastrik I, D'Eustachio P, Schmidt E, et al. Reactome: a knowledge base of biologic pathways and processes. Genome Bio/ 2007;8:R39.

29. Smith KA, Noël E, Thurlings I, Rehmann H, Chocron S, Bakkers J. Bmp and nodal independently regulate lefty 1 expression to maintain unilateral nodal activity during left-right axis specification in zebrafish. PLoS Genet 2011;7:e1002289.

30. Bisgrove BW, Morelli SH, Yost HJ. Genetics of human laterality disorders: insights from vertebrate model systems. Annu Rev Genomics Hum Genet 2003;4:1-32.

31. Chocron S, Verhoeven MC, Rentzsch F, Hammerschmidt M, Bakkers J. Zebrafish Bmp4 regulates left-right asymmetry at two distinct developmental time points. Dev Bio/ 2007;305:577-588.

32. Gavrilov S, Lacy E. Genetic dissection of ventral folding morphogenesis in mouse: embryonic visceral endoderm-supplied BMP2 positions head and heart. Curr Opin Genet Dev 2013;23:461-469.

33. Zhao GQ. Consequences of knocking out BMP signaling in the mouse. Genesis 2003:35:43-56.

34. Genecards: Weizmann Institute of Science. 2013. http://www.genecards.org/ cgi-bin/carddisp.pl?gene=FGF12. Accessed on 3 April, 2013.

35. Mizoguchi T, Izawa T, Kuroiwa A, Kikuchi Y. Fgf signaling negatively regulates Nodal-dependent endoderm induction in zebrafish. Dev Biol 2006;300:612622.

36. Hong SK, Dawid IB. FGF-dependent left-right asymmetry patterning in zebrafish is mediated by ler2 and Fibp1. Proc Natl Acad Sci USA 2009;106:2230-2235.

37. Lenhart KF, Holtzman NG, Williams JR, Burdine RD. Integration of nodal and BMP signals in the heart requires FoxH1 to create left-right differences in cell migration rates that direct cardiac asymmetry. PLoS Genet 2013;9:e1003109.

38. Cha SW, McAdams M, Kormish J, Wylie C, Kofron M. Foxi2 is an animally localized maternal mRNA in Xenopus, and an activator of the zygotic ectoderm activator Foxi1e. PLoS One 2012;7:e41782.

39. Komuro I, Schalling M, Jahn L, et al. Gtx: a novel murine homeobox-containing gene, expressed specifically in glial cells of the brain and germ cells of testis, has a transcriptional repressor activity in vitro for a serum-inducible promoter. $E M B O$ J 1993:12:1387-1401.

40. Duclos A, Charbonnier F, Chambon P, et al. Pitfalls in the use of DGV for CNV interpretation. Am J Med Genet A 2011;155A:2593-2596. 